Article

\title{
Experimental Investigation of the Performance and Spray Characteristics of a Supersonic Two-Phase Flow Ejector with Different Structures
}

\author{
Shizhen $\mathrm{Li}^{1}{ }^{1}$, Wei $\mathrm{Li}^{1}$, Yanjun Liu ${ }^{1,2}$, Chen $\mathrm{Ji}^{1}$ and Jingzhi Zhang ${ }^{3, *}$ \\ 1 Institute of Marine Science and Technology, Shandong University, Qingdao 266237, China; \\ lishizhen@sdu.edu.cn (S.L.); LiweiIMST@mail.sdu.edu.cn (W.L.); lyj111@sdu.edu.cn (Y.L.); \\ jich@zju.edu.cn (C.J.) \\ 2 School of Mechanical Engineering, Shandong University, Jinan 250061, China \\ 3 School of Energy and Power Engineering, Shandong University, Jinan 250061, China \\ * Correspondence: zhangjz@sdu.edu.cn; Tel.: +86-187-6976-6616
}

Received: 14 January 2020; Accepted: 2 March 2020; Published: 4 March 2020

\begin{abstract}
A two-phase flow ejector is an important part of a water mist fire suppression system, and these devices have become a popular research topic in recent years. This paper proposes a supersonic ejector that aims to improve the efficiency of water mist fire suppression systems. The effects of ejector geometric parameters on the entrainment ratio (ER) were explored. The effects of primary flow pressure $\left(P_{\mathrm{P}}\right)$ on the mixing process and flow phenomena were studied by a high-speed camera. The experimental results show that the ER first increases and then decreases with increasing $P_{\mathrm{P}}$. ER increases with increasing ejector area ratio (AR). The $P_{\mathrm{P}}$ corresponding to the maximum ER of ejectors with a different nozzle exit position (NXP) is 3.6 bar. The ejector with an NXP of +1 and AR of 6 demonstrate the best performance, and the ER of this ejector reaches 36.29. The spray half-cone angle of the ejector increases with increasing ER, reaching a maximum value of $7.07^{\circ}$. The unstable atomization half-cone angle is mainly due to a two-phase flow pulsating phenomenon. The pulsation period is $10 \mathrm{~ms}$. In the present study, a general rule that provides a reference for ejector design and selection was obtained through experiments.
\end{abstract}

Keywords: two-phase ejectors; entrainment ratio; ejector structure; spray characteristics; visualization

\section{Introduction}

Fire protection, as an important field of social and public security, is closely related to society and everyone involved in the process of national economic development. With the progress of science and technology, more advanced firefighting equipment has been developed. Water mist fire suppression systems are among these technologies, and research on ejectors, the core component of a water mist fire suppression system, is very significant. An ejector is a type of fluid machinery that uses working fluids with high and low pressures to realize energy conversion and application [1,2]. Two-phase ejectors have been widely used in chemical, biochemical, petroleum, refrigeration, and other industries because of the simple structure, low cost, superior two-phase contact, and heat transfer characteristics of these ejectors [3]. Compared with theoretical and numerical methods, the experimental method can obtain reliable data.

Since the invention of the ejector, many investigations have been conducted to improve the efficiency of this device. A large number of studies have shown that a proper ejector can significantly improve the performance of a system. Zhou et al. [4] introduced a mixer-ejector, and they put forward a minimum injection pressure of $0.25 \mathrm{MPa}$ to ensure the methane/air mixer-ejector works normally. Chandra and Ahmed [5] as well as Hu et al. [6] concluded that a change in the geometric parameters 
of an ejector has a significant impact on the ejector performance. Taleghani et al. [7] proposed that the ejector's performance is affected by the maximum transiting exergy efficiency around the critical point. Nakagawa et al. [8] analyzed the influence of the mixing chamber length on the ejector system performance through experiments. The results showed that the mixing chamber lengths of 5 and 15 mm yielded the lowest and highest ejector efficiency, respectively. Liu et al. [9] and Dong et al. [10] studied a two-phase flow ejector with variable geometry and found that the efficiency decreased with decreasing ejector area ratio. Yan et al. [11] regarded the two ejectors as a whole and used two-dimensional Computational Fluid Dynamics (CFD) and experimental methods to explore the effect of key parameters on its performance. Ultimately, these researchers proposed that the parameter with the greatest effect on the entrainment ratio is the area ratio (AR). Thongtip and Aphornratana [12] found that with increasing distance between the nozzle and the entrance of the mixing chamber, the performance of the ejector improved slightly. Li et al. [13] proposed that the exit position of nozzle has a significant effect on the performance of the refrigeration system. When the exit position of the nozzle moved from 0 to $7.5 \mathrm{~mm}$, the entrainment ratio changed from 6.25 to 8.33; that is, the entrainment ratio increased with increasing nozzle exit position (NXP). Wang et al. [14] studied the effect of surface roughness on the entrainment ratio. Cui et al. [15] also discussed the effects of manufacturing deviations on cavitation and jet flow. The ejection ratio decreased gradually with increasing surface roughness. Therefore, the nozzle should be polished as much as possible to ensure extinguishing efficiency.

Although the structure of the ejector is relatively simple, the internal mechanism of the ejector is complex due to the complexity of the supersonic flow field [16]. The fluid flow process of the ejector includes the interaction of the shock wave, boundary layer, and shear layer. Thus far, the flow mechanism of a two-phase ejector has not been fully understood. This knowledge gap leads to the adoption of semitheoretical and semiempirical methods in ejector design, which results in a series of problems such as poor performance, unstable operation, and serious discrepancies between design efficiency and practice. It is difficult to explain the complex flow phenomena in the two-phase flow ejector by means of the analysis of quantitative results. Therefore, studying the complex mixing process of two-phase flow with a high-speed camera is necessary. Due to the pressure difference between the suction chamber and the secondary flow, the secondary fluid can be entrained in the primary flow in the form of small droplets or bubbles [17]. The phenomenon of spray pulsating exists in all nozzles [18,19]. For two-phase flow atomization, there are obvious fluctuations in the gas-liquid flow coefficient in the vicinity of the nozzle due to the gas phase. Maldonado et al. [20] studied the pressure fluctuation in the nozzle. The authors found that the flow pattern inside the nozzle had a violent effect on the spray pulsation. Lin et al. [21] studied the pulsating frequency and amplitude of the internal pressure of the bubble atomizing ejector. It was found that reducing the nozzle diminishing angle and the length of the spray orifice can effectively reduce the spray pulsating phenomenon. Through experiments, a variety of measuring techniques were used to study the atomization characteristics of ejectors. Most of these experiments focused on the macroscopic characteristics of atomization (spray angle, breakup length of liquid film, and droplet size distribution) [22-24]. Jia et al. [25] measured the spray penetration using a high-speed camera and pointed out that the cone angle increased with pressure. Esfarjani and Dolatabadi [26] found that by increasing the aeration level, the mixing between the gas and liquid is enhanced, and the flow structure near the nozzle exit changes from slug flow to co-annular flow. Zhu et al. [27] employed $\mathrm{CO}_{2}$ as the working medium to visualize the flow process inside the ejector. It was pointed out that if the expansion angle of the nozzle outlet is too large, the primary flow might occupy part of the flow area of the secondary fluid, decreasing the entrainment ratio.

The performance of the ejector directly affects the efficiency of fire extinguishing. It is necessary to study the performance of the ejector and understand the flow phenomena inside the ejector. Although many numerical studies have been carried out on the entrainment ratio and velocity field of supersonic ejectors with single-phase flows, experimental studies on two-phase flow ejectors used in water mist fire suppression systems are still lacking. The influence of different ARs and different NXPs on the ejector entrainment ratio was studied experimentally. The influence of the primary flow pressure on the 
entrainment ratio and flow characteristics in the fire suppression system was investigated. The effect of the primary mass flow rate on the spray half cone-angle was obtained by studying the atomization state of a visualized two-phase ejector. To further deepen the understanding of the atomization state of the ejector, the pulsating characteristics of the ejector was analyzed, which can provide guidance for the structure design and selection of working conditions for a two-phase flow ejector suitable for a water mist fire extinguishing system.

\section{Experimental Setup}

\subsection{Experimental System}

As shown in Figure 1, the experimental setup of the two-phase flow ejector is mainly composed of a gas supply system (air compressor, gasholder, and pressure control valve), a liquid supply system (ball valve, elevated water tank, pump, and storage tank), visualized ejectors with different structural parameters, and a measurement system (high-speed camera, vortex flowmeter, turbine flowmeter, data acquisition card, and computer). Water was employed as the liquid working medium, and compressed air was employed as the gas working medium. The gas pressure was regulated within the range of 0-6 bar, while a standard atmospheric pressure was maintained for water. The experiment was carried out at room temperature.

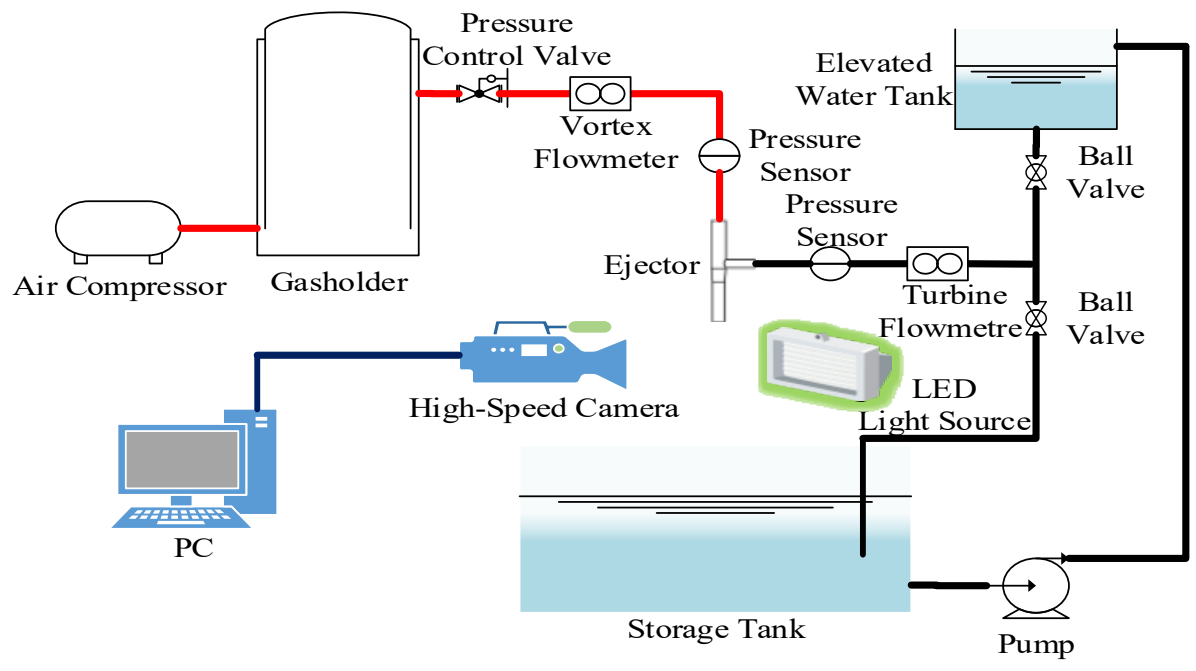

(a)

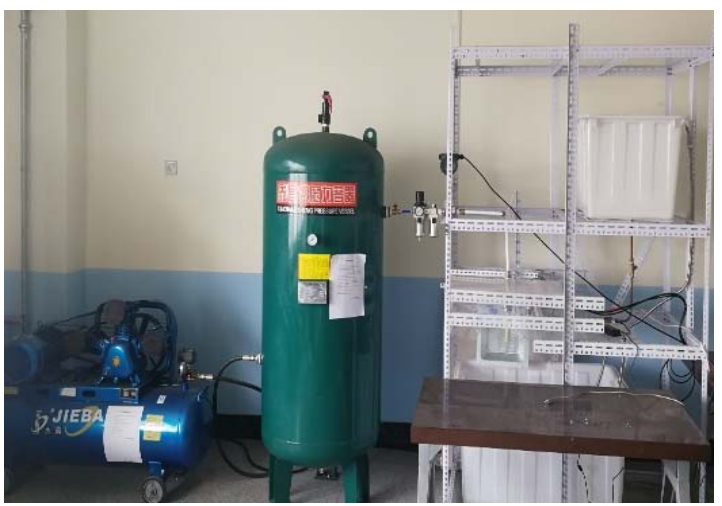

(b)

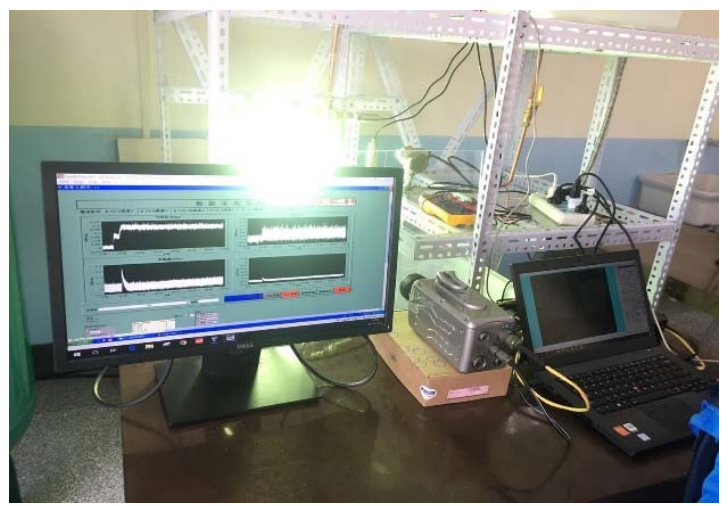

(c)

Figure 1. Schematic of the experimental setup. (a) the experimental setup; (b) the gas and liquid supply system; (c) the measurement system. 
A reciprocating air compressor with a power of $7.5 \mathrm{~kW}$ was selected to provide the power of the primary flow. The volume of the gasholder used was $600 \mathrm{~L}$ to fulfil the role of gas storage, buffer, and stabilization. The gas pressure $\left(P_{\mathrm{P}}\right)$ was controlled by a pressure control valve and measured by a diffused-silicon pressure transmitter with $0.2 \%$ error. To obtain relatively dry and stable air, a pressure control valve with a filtering accuracy of $5 \mu \mathrm{m}$ was used. The gas flow rate $\left(Q_{G}\right)$ and liquid flow rate $\left(Q_{\mathrm{L}}\right)$ were measured before gas-liquid mixing. A DN15 vortex flowmeter with an accuracy of $0.5 \%$ was used to measure $Q_{\mathrm{G}}$, and a DN4 turbine flowmeter with an accuracy of $0.5 \%$ was used to measure $Q_{\mathrm{F}}$. A Phantom high-speed camera $(1600 \times 1200$ pixels, $1000 \mathrm{fps})$ was employed to record the mixing flow pattern and atomization characteristics of the two-phase flow, and an LED lamp (200 W) was used as a constant light source to ensure high image quality. A filter was attached to the LED lamp to uniformly distribute the light and adjust the brightness. The LED lamp and the high-speed camera were installed on the same line. The ejector was installed vertically and placed between the light source and the high-speed camera. The high-speed camera was connected to a computer with an Ethernet cable, and image acquisition software was employed to collect and store the images. The flowmeter and pressure sensor signals were transferred to the computer data acquisition software through an Advantech data acquisition card, which had a sampling frequency of $200 \mathrm{KS} / \mathrm{s}$.

To improve data stability, data acquisition was started in the $5 \mathrm{~s}$ after the ejector began spraying. In the two-phase flow ejector experimental platform, the two-phase mass flow rates and entrainment ratios of different ejector structures were studied, and the atomization characteristics and mixing state of the two-phase flow were analyzed, providing a basis for the design of a two-phase flow ejector.

\subsection{Geometry of the Two-Phase Ejector}

The geometry of the supersonic two-phase flow ejector is shown in Figure 2. The ejector consists of five parts: the primary nozzle, suction chamber, constant-pressure mixing chamber, constant-area mixing chamber, and diffuser.

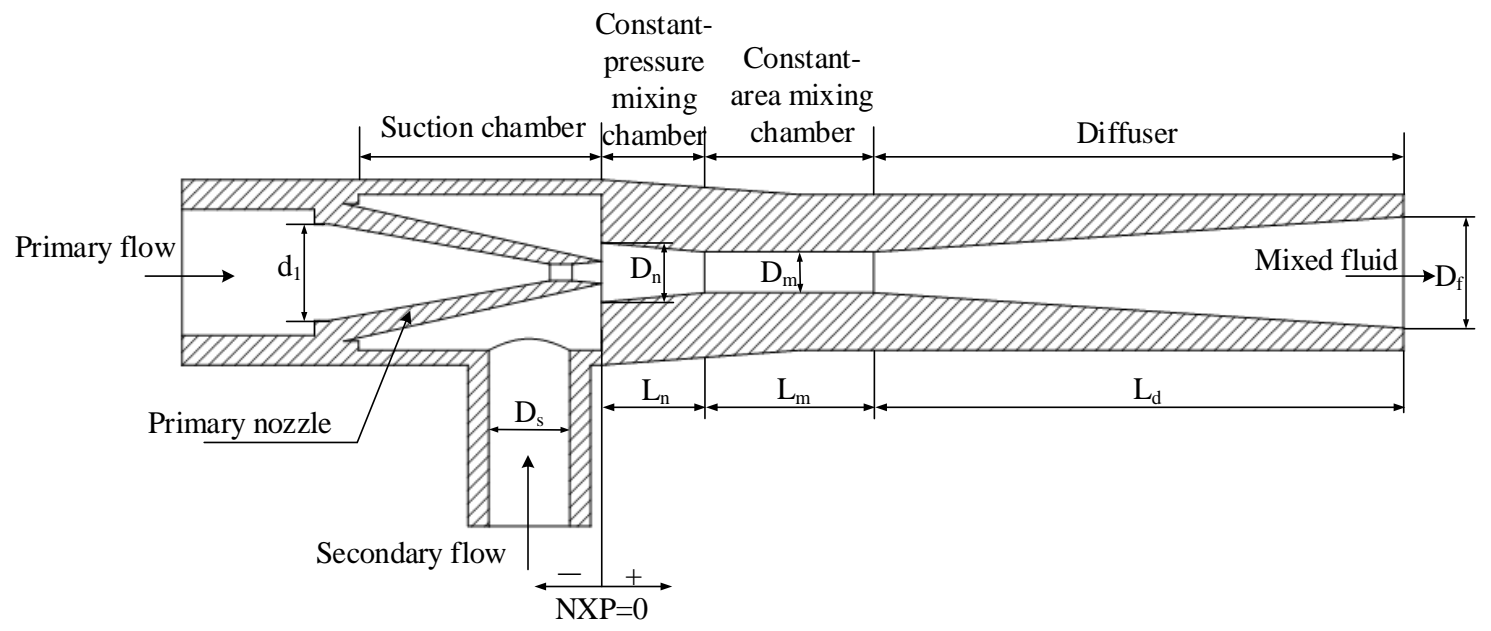

Figure 2. Structure of the two-phase flow ejector.

The whole ejector was modelled by Solidworks and was processed using 3D printing technology, providing more precise control of the ejector size and a smoother surface than traditional methods. To facilitate the image capture of the high-speed camera and ensure the visualization of the experiment, a water resistant, transparent photosensitive resin with a smooth surface was selected. The main geometric parameters of the injector employed in the experiment are shown in Table 1. 
Table 1. Main geometric parameters of the ejector.

\begin{tabular}{cc}
\hline Geometric Parameters & Value (Unit, $\mathbf{m m}$ ) \\
\hline Diameter of primary nozzle inlet $\left(d_{1}\right)$ & 13 \\
Diameter of constant-pressure mixing chamber inlet $\left(D_{\mathrm{n}}\right)$ & 8 \\
Diameter of constant-area mixing chamber $\left(D_{\mathrm{m}}\right)$ & 5.5 \\
Diameter of diffuser outlet $\left(D_{\mathrm{f}}\right)$ & 15 \\
Diameter of secondary flow chamber $\left(D_{\mathrm{s}}\right)$ & 11 \\
Length of primary nozzle $\left(L_{\mathrm{p}}\right)$ & 37 \\
Length of constant-pressure mixing chamber $\left(L_{\mathrm{n}}\right)$ & 14 \\
Length of constant-area mixing chamber $\left(L_{\mathrm{m}}\right)$ & 23 \\
Length of diffuser $\left(L_{\mathrm{d}}\right)$ & 72 \\
Area ratio (AR) & $2,3,4,5,6$ \\
Nozzle exit position $(\mathrm{NXP})$ & $-1,0,+1$ \\
\hline
\end{tabular}

The high-pressure primary flow expands after passing through the nozzle throat and is accelerated to supersonic speed. The pressure decreases greatly, becoming lower than the secondary flow pressure. Therefore, the pressure difference between the flows entrains the secondary flow into the mixing chambers and accelerates the flow to the sound velocity in the mixing chamber. The primary and secondary flows are accompanied by intense kinetic energy exchange. At this time, impact, shear, tension, and ultrasonic resonance occur, breaking up the liquid. With the rapid change in the pressure of the mixed flow, the final shock wave occurs at the end of the mixing chamber, and further pressure recovery is achieved in the diffuser. Next, the flow is ejected from the outlet of the ejector to realize the effective atomization of water. Then, the droplets begin to diffuse, and a conical spray is formed downstream of the ejector exit.

Generally, the entrainment ratio (ER) is one of the key parameters for evaluating the performance of the ejector. The ER is defined as

$$
\mathrm{ER}=\frac{\dot{m}_{\mathrm{s}}}{\dot{m}_{\mathrm{p}}}
$$

where $m_{\mathrm{p}}$ is the primary flow mass flow rate $(\mathrm{g} / \mathrm{s})$ and $m_{\mathrm{s}}$ is the secondary flow mass flow rate $(\mathrm{g} / \mathrm{s})$. The greater the ER is, the better the ejector performance is.

As illustrated in Figure 3, ER is closely related to the back pressure $\left(P_{\mathrm{b}}\right)$. The ejector operational modes can be classified into three types according to the different back pressures: the critical mode, the subcritical mode, and the back flow mode. In the critical mode, with increasing $P_{\mathrm{b}}$, the ER remains unchanged. This state continues to the dividing point between the critical mode and subcritical mode, which is called the critical point. The corresponding back pressure value is called critical back pressure $\left(P_{\mathrm{b}}{ }^{*}\right.$. When $P_{\mathrm{b}}$ continues to increase above $P_{\mathrm{b}}{ }^{*}$, the ejector enters subcritical mode, and the ejector still works but is unstable. At this time, the ER gradually reduces to 0 with further increase of $P_{\mathrm{b}}$, and $P_{\mathrm{b}}$ continues to increase, causing the ejector to enter the back flow mode. In this mode, the ER is always 0 , and the secondary flow cannot enter the ejector when the primary flow emanates from the secondary inlet; i.e., the ejector is dysfunctional. The optimum working condition for the ejector is that when the back pressure is just at $P_{\mathrm{b}}{ }^{*}$; the ejector performance deteriorates rapidly when the ejector is operated in back flow mode [28]. 


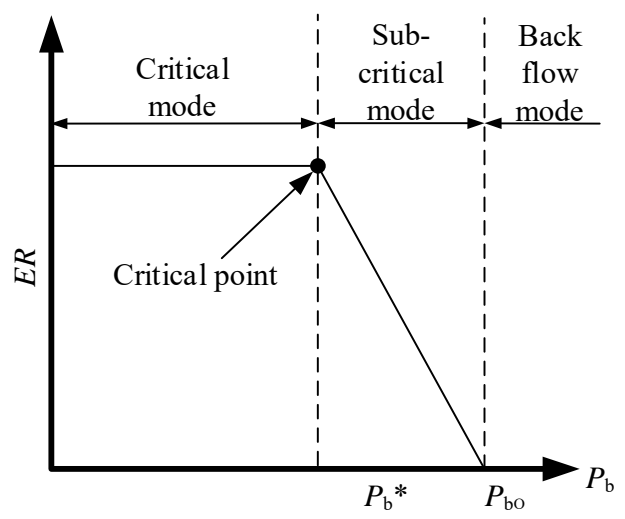

Figure 3. Typical performance curve of a supersonic ejector.

The working conditions and the structure of the ejector are the two most important factors affecting the performance of the ejector. The working conditions include the physical characteristics of the primary and secondary flows, as well as the pressures of both. The ejector structure is mainly determined by the area ratio (AR), the nozzle exit position (NXP), and the length of the constant-area mixing chamber $\left(L_{\mathrm{m}}\right)$, where the AR is defined as the area ratio between the constant-area mixing chamber throat and primary nozzle throat, i.e.,

$$
\mathrm{AR}=\left(\frac{D_{\mathrm{m}}}{D}\right)^{2}
$$

where $D_{\mathrm{m}}$ is the diameter of the constant-area mixing chamber and $D$ is the diameter of the primary nozzle throat.

Figure 2 shows that the magnitude of the NXP is defined as the distance between the primary nozzle and constant-pressure mixing chamber.

The spray half cone-angle $(\theta)$ is an important characteristic parameter describing the spray shape, which can reflect the atomization effect of the ejector. The ejector outlet is defined as the starting point, and the corresponding length of each pixel can be determined based on the ejector diffuser outlet diameter. Figure 4 shows the definition of this angle, and the following atomization field trapezoidal domains are adopted: the trapezoidal upper base is $D_{f}$, the height is $4 D_{f}$, and the spray half cone-angle can be calculated by means of the following,

$$
\theta=\arctan \left(\frac{\mathrm{b}}{4 D_{\mathrm{f}}}\right)
$$

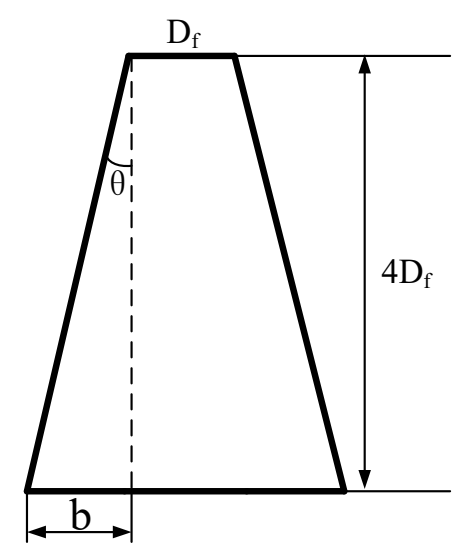

Figure 4. Definition of the spray half cone-angle. 


\section{Results and Discussion}

\subsection{Effect of Area Ratio on Ejector Performance}

The experiment was carried out using ejectors with an NXP of 0 but with different ARs (2-6), mainly to study the effect of the AR on the ejector performance. During the experiment, the $P_{\mathrm{b}}$ was kept unchanged, and the pressure of the primary flow $\left(P_{\mathrm{P}}\right)$ was changed. The pressure adjustment range was $0.4-6$ bar, and a set of data was measured every 0.4 bar. The contrast curve of the ER for different $P_{\mathrm{P}}$, shown in Figure 5, was obtained. Figure 5 shows that the ERs of the different ARs increase with increasing $P_{\mathrm{P}}$, and the ERs tend to first increase and then decrease after a peak value is reached. Wang et al. [29] reached a similar conclusion by simulation calculations. Before the ER reaches the maximum value, an increase in $P_{P}$ results in an increase in the ER, indicating that the ejector is in a subcritical mode at this time, and an increase in the pressure in the subcritical mode increases the kinetic energy of the working fluid. The ability to entrain the primary flow and the ability of the fluid to overcome the back pressure of the ejector are enhanced, thereby facilitating an increase in the ER. The maximum ERs of the five different structure ejectors are 1.32, 12.98, 16.46, 23.08, and 28.07, respectively. After the ER reaches the maximum value, the ER decreases as $P_{P}$ increases, indicating that the ejector is in the critical mode. Obviously, the maximum ER is the boundary between the critical mode and the subcritical mode. The ejector achieves the best performance at this time, which is the optimal working condition we need for the ejector; although the AR2 and AR3 ejectors can work under higher pressure and the AR5 and AR6 ejectors can work under lower pressure, the ERs at these pressures are not the most ideal values; that is, we need to adjust the $P_{P}$ to reach the specified value for the different structures of the ejector. By comparing the ER curves of different AR ejectors, it can be found that the larger the AR is, the larger the ER is in the critical mode; the subcritical mode shows similar trends, but because of the extreme instability of the subcritical mode, the performance of the AR5 and AR6 ejectors when $P_{P}$ is lower than 2.4 bar is not superior to that of other structures. The ER cannot be increased simply by increasing the $P_{\mathrm{P}}$. The working range of $P_{\mathrm{P}}$ should also be fully considered when selecting the ejector structure, especially for a water mist fire suppression system.

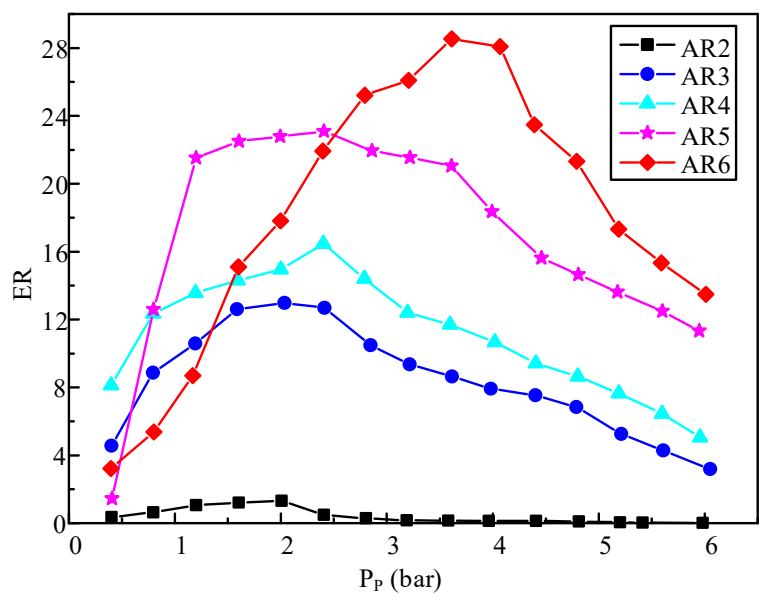

Figure 5. Effects of primary flow pressure on ejector performance at various area ratios (ARs).

To investigate why the ER decreases when the $P_{\mathrm{P}}$ is greater than a certain fixed value, we compiled a figure comparing the influence of $P_{P}$ on the gas and liquid mass flow rates of the same AR ejector, as shown in Figure 6. The figure shows that there are similarities in the ejector gas and liquid mass flow curves of different ARs: the primary flow mass flow rate $\left(m_{\mathrm{p}}\right)$ gradually increases with increasing $P_{\mathrm{P}}$, and the secondary flow mass flow rate $\left(m_{\mathrm{S}}\right)$ has a tendency to first increase and then decrease as $P_{\mathrm{P}}$ increases. Combining Figure 6 with the definition of the ER, it can be inferred that the trend of $m_{\mathrm{s}}$ is the main factor behind the trend of the ER. On the other hand, the gas and liquid mass flow 
rate curves also have differing characteristics: for different $A R$ ejectors, the $P_{\mathrm{P}}$ values corresponding to the maximum $m_{\mathrm{s}}$ differ, gradually increasing with increasing AR. Comparing the above curves, it can be found that the maximum value of $m_{\mathrm{S}}$ increases with increasing AR from 2 to 5 , with values of $2.26,13.29,15.46$, and $18.07 \mathrm{~g} / \mathrm{s}$, respectively, but the maximum $m_{\mathrm{s}}$ of the AR6 ejector is only 14.96 $\mathrm{g} / \mathrm{s}$, and the $m_{\mathrm{s}}$ decreases extremely slowly. In contrast, $m_{\mathrm{p}}$ increases with increasing $P_{\mathrm{p}}$ for all cases. The increase in $P_{\mathrm{P}}$ increases the difference between $P_{\mathrm{p}}$ and the outlet pressure, enhancing the ability of the secondary flow to overcome the $P_{\mathrm{b}}$ of the ejector and resulting in an increase in the secondary flow into the suction chamber, which is sufficiently mixed and increases the ER. When the working pressure exceeds the critical value, there will be more resistance at the outlet of ejector, the flow capacity of the ejector has reached the maximum, constant-pressure and constant-area mixing chamber is chocked, the secondary flow is blocked, no matter how much $P_{\mathrm{P}}$ increases, the secondary flow will not increase as before. When ER gets a larger value, it means that the primary flow with less consumption can absorb more secondary flow, thereby the number of broken droplets increases, and the fire extinguishing performance is better.

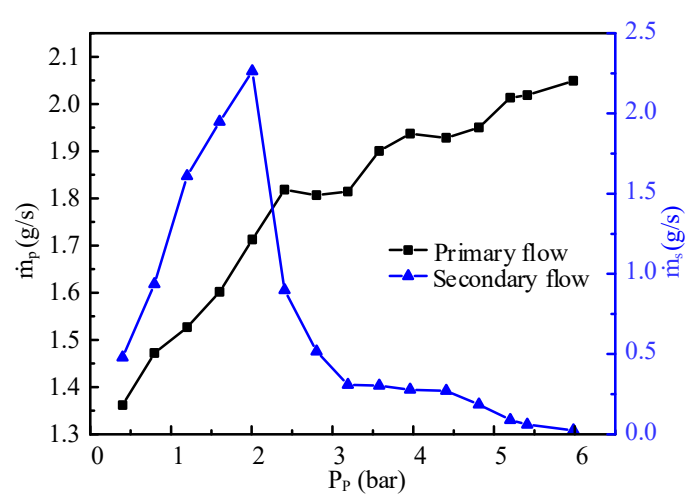

(a)

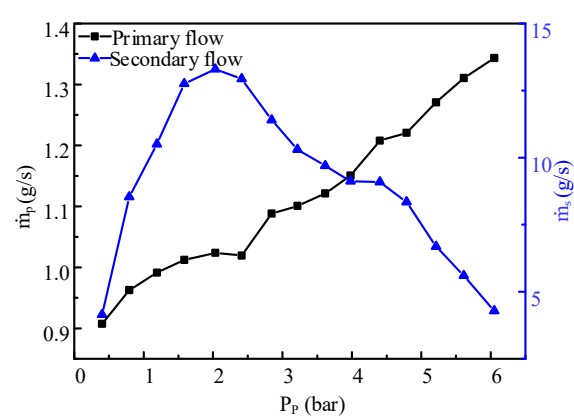

(b)

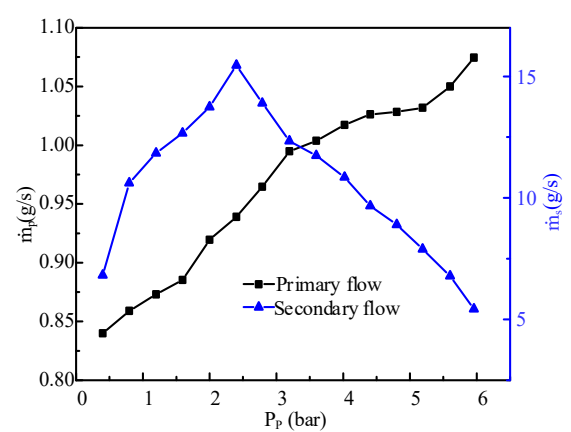

(d)

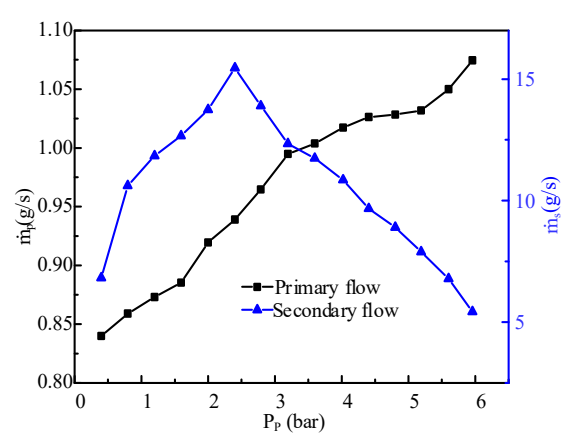

(c)

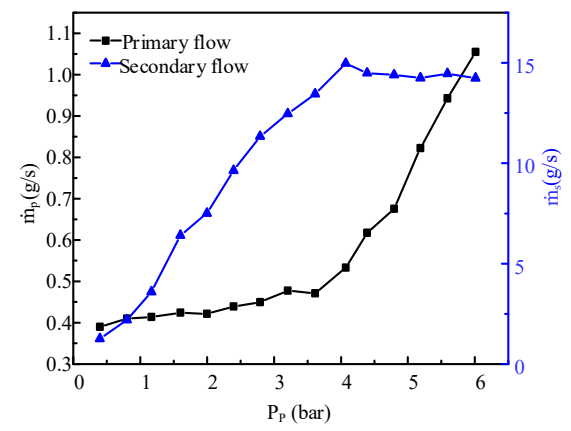

(e)

Figure 6. Effects of primary flow pressure on mass flow rates at various $A R s$. (a) $A R=2 ;(\mathbf{b}) A R=3$; (c) $\mathrm{AR}=4 ;(\mathrm{d}) \mathrm{AR}=5 ;(\mathbf{e}) \mathrm{AR}=6$. 


\subsection{Effect of Nozzle Exit Position on Ejector Performance}

To study the relationships between the other important structural parameters of the ejector, the NXP, and the ER, experiments were carried out using ejectors with different NXPs $(-1,0,+1)$ to maintain the ejector AR at 6 and keep the back pressure $P_{\mathrm{b}}$ constant. The independent variable is still $P_{\mathrm{P}}(0.4-6 \mathrm{bar})$, and the measured $P_{\mathrm{P}}$ data have an interval of 0.4 bar. A comparison chart of the effects of $P_{\mathrm{P}}$ on the ejector performance at different NXPs is shown in Figure 7. The figure shows that the ERs of the ejectors with different NXPs increase with increasing $P_{\mathrm{P}}$. The ejector achieves the best performance when the ER reaches the maximum value. This condition is also the optimum working condition we need for the ejector. Figure 7 shows that the closer the primary nozzle of the AR6 ejector is to the constant-pressure mixing chamber, the larger the ER is, but a greater distance is not desirable because an increase in the NXP causes the cross-sectional area at the inlet of the constant-pressure mixing chamber to decrease, affecting the intake of the secondary flow. Dong et al. [30] also reached a similar conclusion. They concluded that the ejector has an optimum "effective area" at different NXPs, which might affect the "entrained duct" formed by the constant-pressure mixing chamber and primary nozzle outlet. Although the NXPs of the ejectors are at different distances and the maximum ERs are different, the optimum ERs correspond to the same primary flow pressure of $3.6 \mathrm{bar}$, indicating that the optimum $P_{\mathrm{P}}$ of ejectors with the same ARs is independent of NXPs. Therefore, when selecting the structure parameters of the ejector, the optimal $P_{\mathrm{P}}$ range can be adjusted by the difference between the ARs, and the overall performance of the ejector can be improved by adjusting the distance of the NXP to improve the efficiency of the water mist fire suppression system.

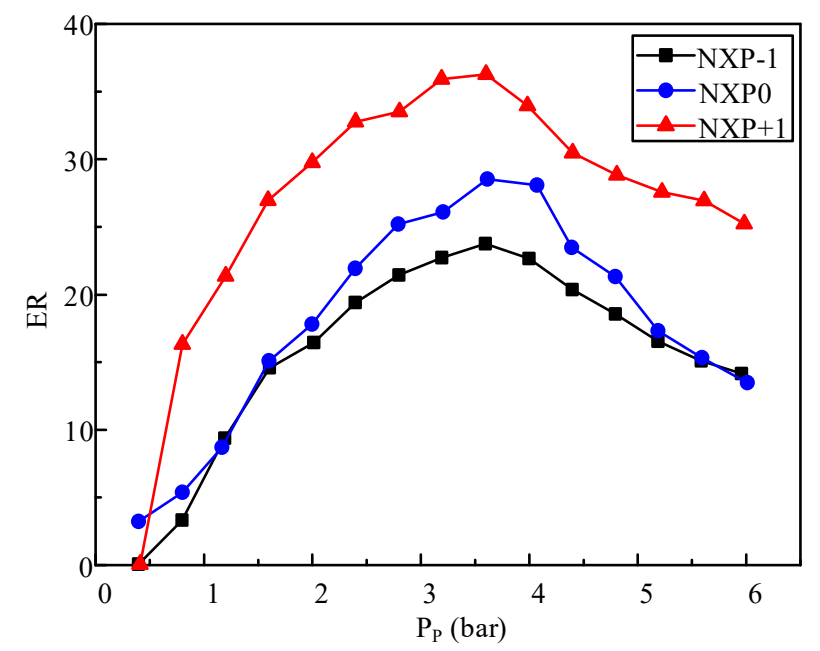

Figure 7. Effects of primary flow pressure on ejector performance at different nozzle exit positions (NXPs).

To further explain the phenomenon and the law disclosed in Figure 7, a comparison chart of the effects of $P_{\mathrm{P}}$ on the mass flow rates at different NXPs was obtained, as shown in Figure $8 . m_{\mathrm{p}}$ always increases with increasing $P_{\mathrm{P}}$, with the increase of the $P_{\mathrm{P}}$, the mixing boundary layer in the ejector is expanding, from the inlet section of the constant-pressure to the constant-area mixing chamber; the expansion of the boundary layer results in increase of the $m_{\mathrm{s}}$. As the increase of the $m_{\mathrm{p}}$ is greater than the increase of the $m_{\mathrm{s}}$, the ER is generally increased. Once beyond the maximum operating ER, that is, at a working fluid pressure greater than 3.6 bar, the growth rate significantly increases, while $m_{\mathrm{S}}$ shows an overall trend of first increasing and then decreasing with increasing $P_{\mathrm{P}}$. The curve slope of $m_{\mathrm{S}}$ is small only during the reduction process; $m_{\mathrm{s}}$ increases gradually, and the secondary flow, dispersed in the mixing chamber uniformly by the action of shear force, drives the entraining flow rate to the maximum. Then, the momentum of the jet at the inlet of the mixing chamber is low, resulting in a decrease in $m_{\mathrm{s}}$. Han et al. [31] gave an explanation from the point of view of simulation: a reduction in 
NXP leads to insufficient mixing and energy exchange processes. The interaction of the two fluids leads to excessive contact between the mixed fluid and the wall surface, gradually increasing the back pressure gradient and generating a vortex. Combined with the definition of the ER, the trend of $m_{\mathrm{S}}$ is to first increase and then decrease, and the rate of increase of $m_{\mathrm{p}}$ is the main reason for the abovementioned regularity of the ER. It can be clearly seen from the comparison chart that the closer the primary nozzle is to the side of the constant-area mixing chamber, the higher the maximum $m_{\mathrm{S}}$ is, with values of $13.51,14.97$, and $19.86 \mathrm{~g} / \mathrm{s}$ for NXPs of $-1,0$, and +1 , respectively. These changes in the gas and liquid mass flows are the main reasons for the abovementioned variations in the ER.

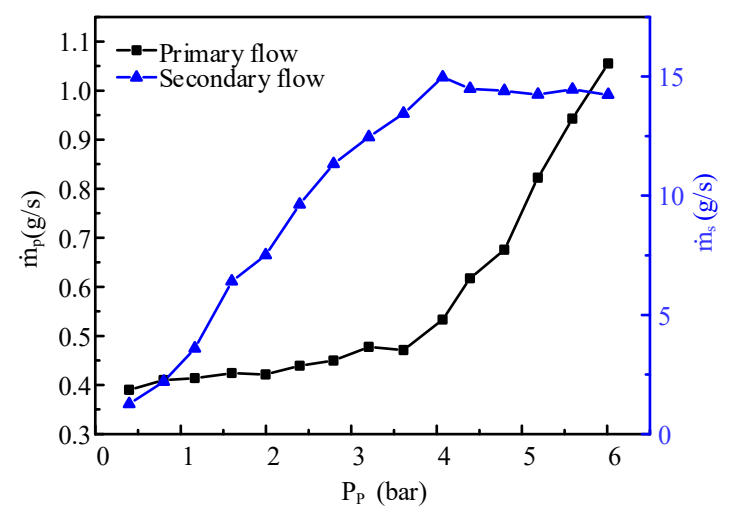

(a)

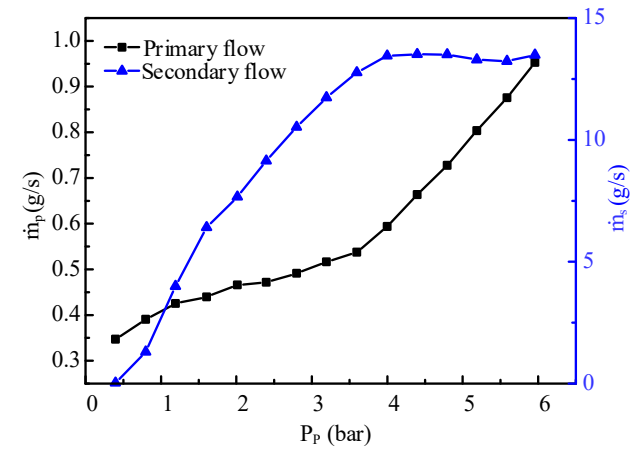

(b)

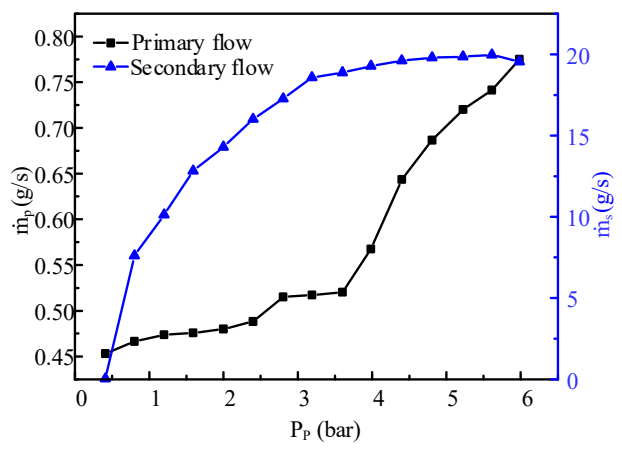

(c)

Figure 8. Effects of primary flow pressure on mass flow rates at different NXPs. (a) NXP = 0; (b) NXP = $-1 ;(\mathbf{c}) \mathrm{NXP}=+1$.

\subsection{Atomization Characteristics with Different Primary Flow Pressures}

Figure 9 shows atomization pictures at different $P_{\mathrm{P}}$. The experiment employs an ejector with an AR of 6 and NXP of +1 to ensure that the ejector has the highest ER compared to those of the other structural ejectors. When the $P_{\mathrm{P}}$ is lower than 0.8 bar, the liquid crushing and atomization effect is not obvious, the spray half cone-angle $(\theta)$ is basically $0^{\circ}$, and a large amount of water mist appears at the outlet of the ejector when the $P_{\mathrm{P}}$ is increased to 1.2 bar. We define this moment as the starting moment of the ejector atomization process. With further increase of $P_{\mathrm{P}}$, it can be clearly observed that after the secondary flow has undergone the combined action of the full mixing with, and the shearing force of, the primary flow, the separation and crushing effect of the droplets become increasingly obvious, and the number of droplets increases significantly in the spray zone. The atomization effect is optimal up to $3.6 \mathrm{bar}$, and the droplet diameter is reduced to a minimum. At this time, the ejector is at the optimal working condition, corresponding to the boundary point between the abovementioned subcritical mode and the critical mode. When the $P_{\mathrm{P}}$ is greater than $3.6 \mathrm{bar}$, the atomization effect is weakened; larger diameter droplets can be observed at 5.6 and 6.0 bar. Zhang Xin et al. [32] named this 
phenomenon a hydraulic flip phenomenon. It is believed that a small amount of air is drawn into the ejector due to the internal pressure difference in the ejector, resulting in a hydraulic flip phenomenon. At this point, the liquid mass flow rate decreases; it is also known from the above investigation that the ER decreases, and the kinetic energy loss caused by the internal shear friction of the ejector increases, so the atomization effect is weakened.
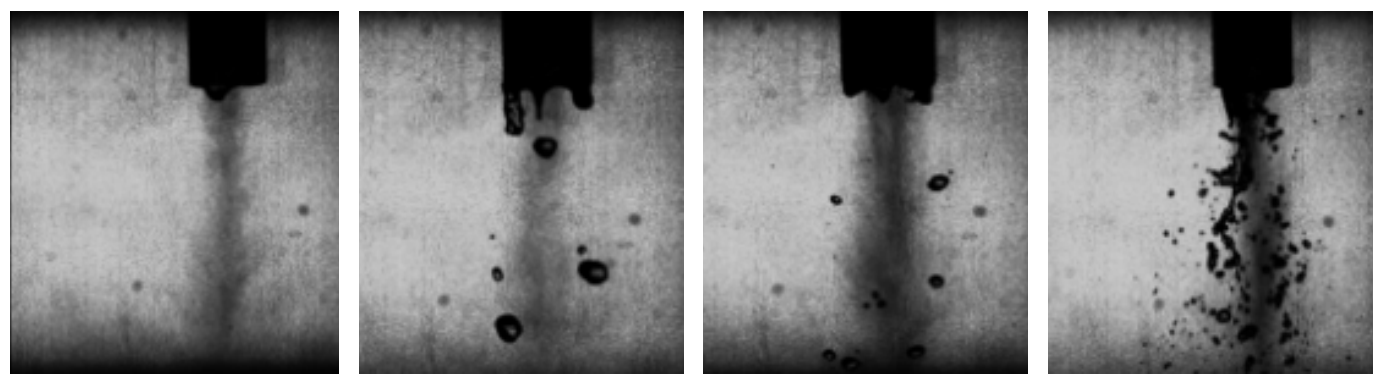

(a)

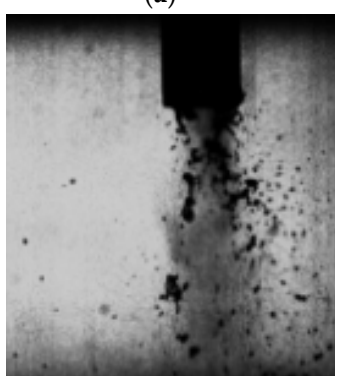

(e)

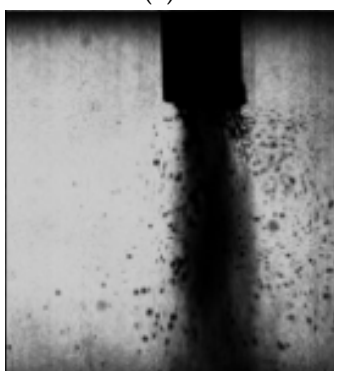

(i)

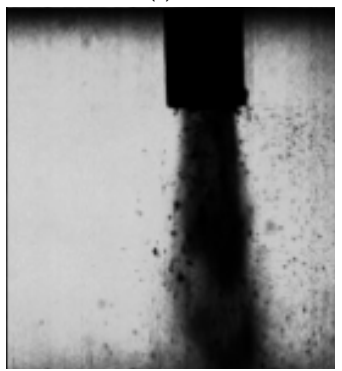

(m) (b)

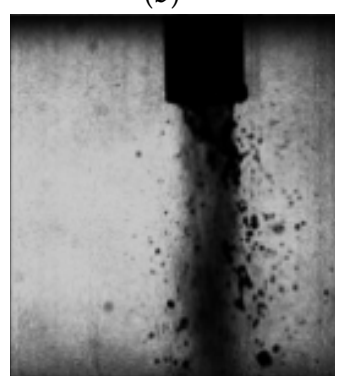

(f)

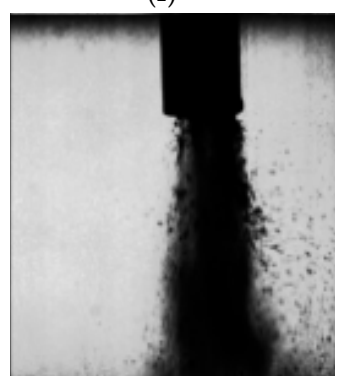

(j)

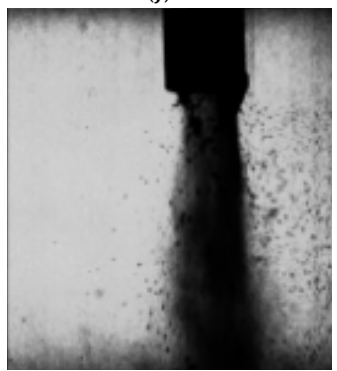

(n) (c)

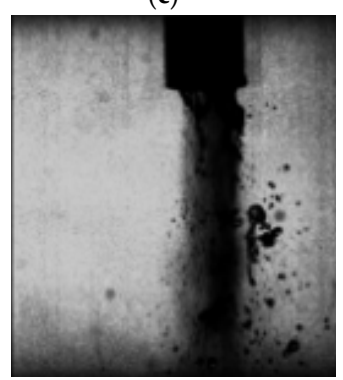

(g)

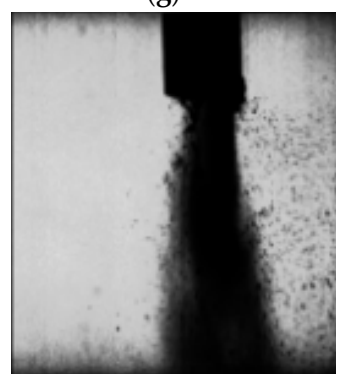

(k)

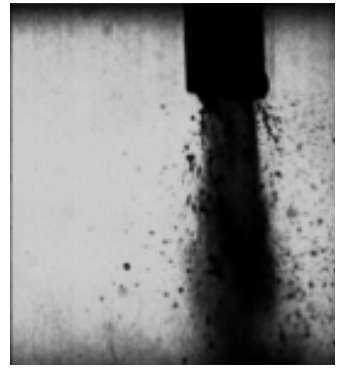

(o) (d)

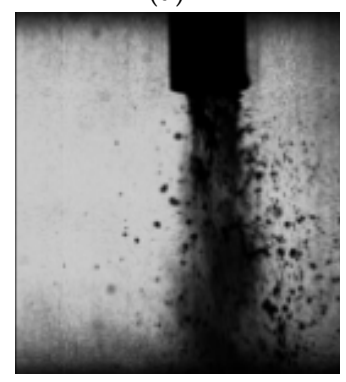

(h)

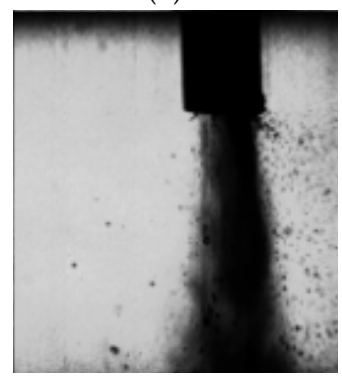

(1)

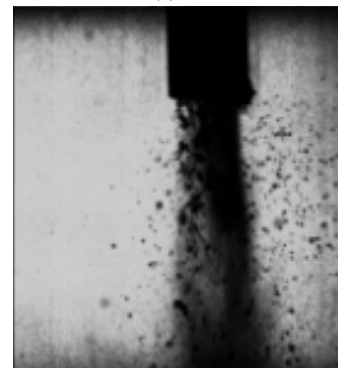

(p)

Figure 9. Evolution of atomization for ejector with different primary flow pressures. (a) 0 bar; (b) 0.4 bar; (c) 0.8 bar; (d) 1.2 bar; (e) 1.6 bar; (f) 2.0 bar; (g) 2.4 bar; (h) 2.8 bar; (i) 3.2 bar; (j) 3.6 bar; (k) 4.0 bar; (1) 4.4 bar; (m) 4.8 bar; (n) 5.2 bar; (o) 5.6 bar; (p) 6.0 bar. 
The size of the spray half cone-angle depends primarily on the viscosity of the liquid, the surface tension, and the degree of turbulence in the jet. The spray half cone-angle is one of the important indexes reflecting the atomization degree, and larger spray half cone-angle is the guarantee of great fire extinguishing performance. To explore the relationship between the spray half cone-angle and the mass flow rate of the primary flow, the color picture was first converted into a grey scale image, and the picture was denoised to eliminate the influence of unrelated interference. Due to the instability of the two-phase flow, the spray angle fluctuates within a certain range, called the pulsation phenomenon of the ejector, so the calculation of the spray half cone-angle was performed on 200 ejector atomization photographs. Therefore, the spray half cone-angle curves at different $m_{\mathrm{p}}$ and $m_{\mathrm{s}}$, as illustrated in Figure 10, were obtained. The figure clearly shows that before the ER reaches the maximum, the spray half cone-angle increases with increasing $m_{\mathrm{p}}$, reaching a maximum value of $7.07^{\circ}$; subsequently, increasing $m_{\mathrm{p}}$ causes atomization to be suppressed, and the spray half cone-angle gradually decreases. When the ER increases, the gas-liquid mixing is more uniform, which is favorable for forming a spray that is continuously stable and has a large spray half cone-angle. Although a higher $m_{\mathrm{p}}$ has a greater impact on the liquid and the degree of extrusion, a higher $m_{\mathrm{p}}$ is not conducive to the sufficient atomization of the combined gas-liquid phases, and the atomization angle is considerably reduced, so the atomization effect is diminished. When ER gets a larger value, it means that the larger the atomization cone angle, the better the atomization effect, the smaller the droplet diameter, and the better the fire extinguishing performance.

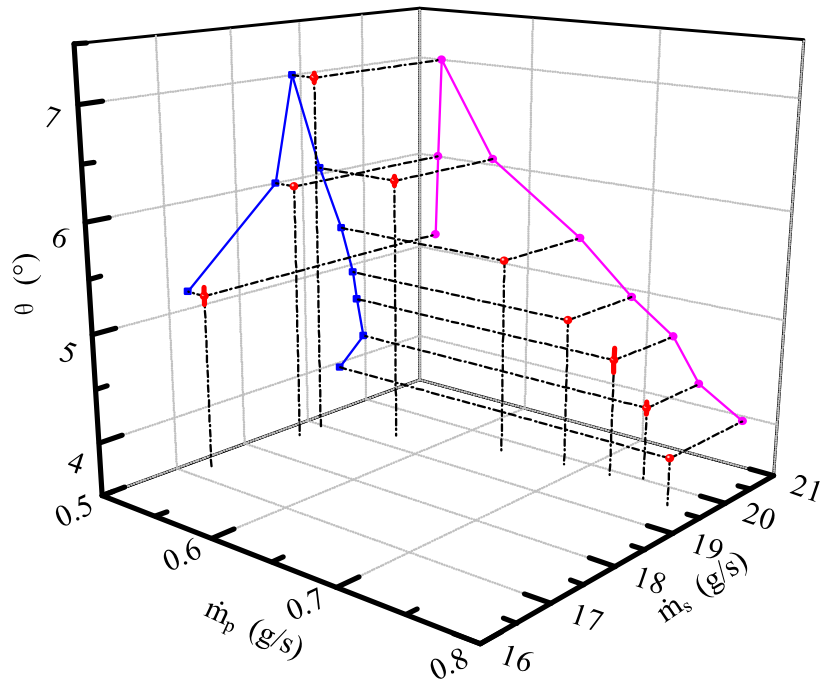

Figure 10. Relationship between the spray half cone-angle and primary and secondary flow mass flow rate.

\subsection{Two-Phase Flow Mixing Pulsating Characteristics}

To investigate the reasons for the instability of the spray half cone angle at different time points, photographs were taken of the phenomenon of gas-liquid pulsation in the suction chamber and diffuser of the ejector under the optimal working conditions. The phenomenon of gas-liquid pulsation exists in all the ejector structures. How to reduce the adverse effect of this pulsation on the efficiency of the ejector is one of the factors that needs to be considered in the structural design of the ejector. The two-phase flow ejector spray pulsation phenomenon is closely related to the gas-liquid two-phase flow pattern (bubble flow, churn flow, and slug flow) in the mixing chamber. Studies have shown that the gas expansion potential energy utilization rate is the highest in bubble flow, and the atomization effect is also better under bubble flow [33]. As shown in Figure 11, even under the same $P_{\mathrm{P}}$, there are significant differences in the mixing pattern of the two-phase flow at different times. The moment when the secondary flow is about to enter the suction chamber is defined as the starting time, that is, the 
starting time $(0 \mathrm{~ms})$ is when the secondary flow reaches the inlet of the suction chamber. The two-phase flow is fully mixed in the suction chamber, and the energy exchange is completed; then, the flow enters the constant-pressure mixing chamber (at the red square) and is sprayed outside the ejector over time. The gas and liquid begin to mix and exchange energy repeatedly after $54 \mathrm{~ms}$ in Figure 11h. At this time, the amount of gas-liquid mixture in the constant-pressure mixing chamber is significantly reduced. The gas-liquid mixing state at $56 \mathrm{~ms}$ in Figure $11 \mathrm{j}$ is similar to the gas-liquid mixing state at $47 \mathrm{~ms}$ in Figure 11a, in order to observe the occurrence of pulsation more intuitively, imageJ software was used to perform binarization processing of the image, so as to obtain the shadow part area in red square and roughly calculate the proportion.

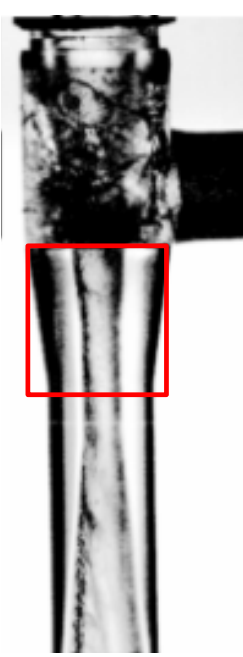

(a)

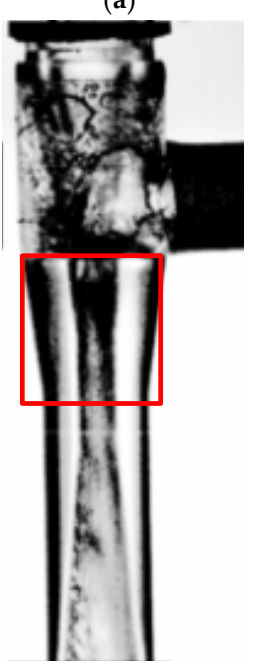

(f)

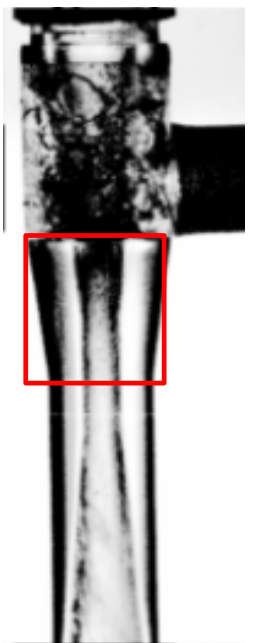

(b)

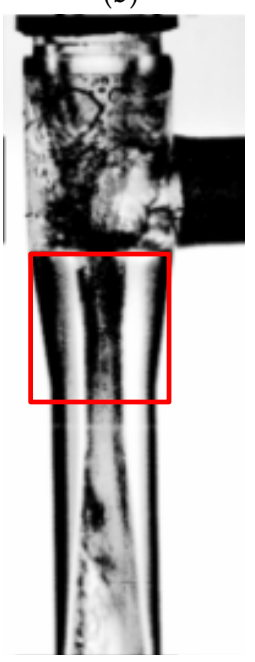

$(\mathrm{g})$

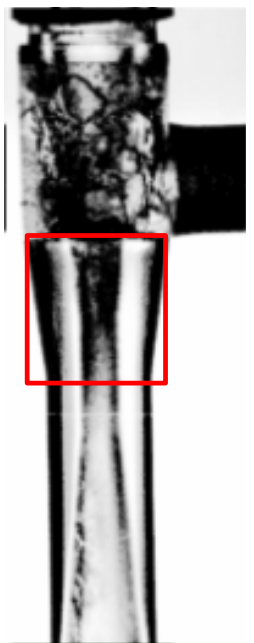

(c)

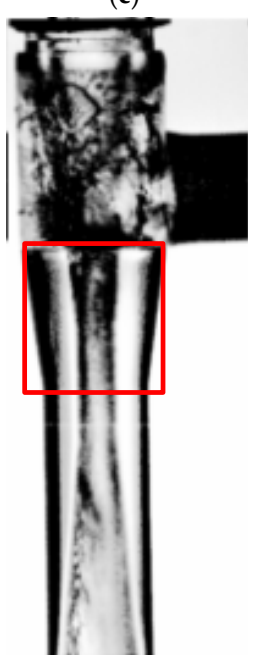

(h)
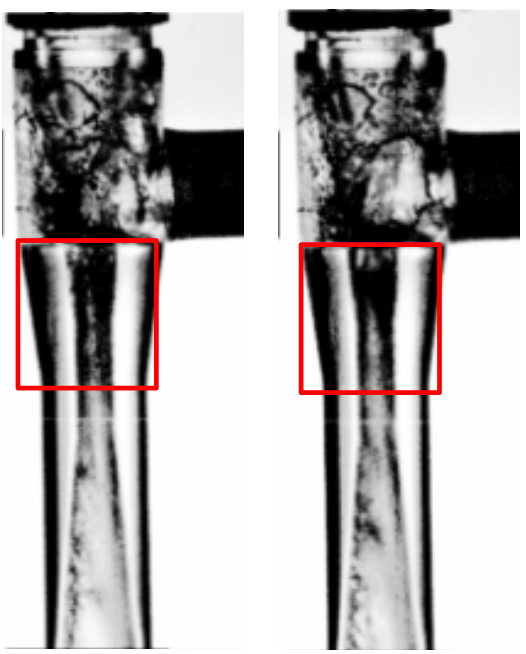

(d)

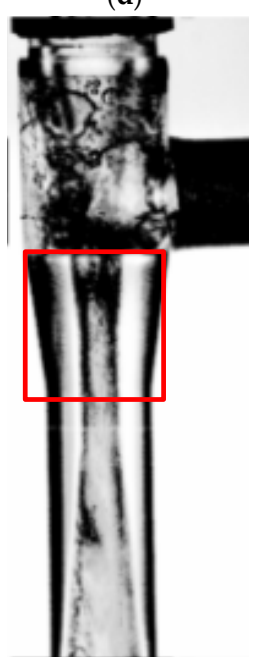

(i)

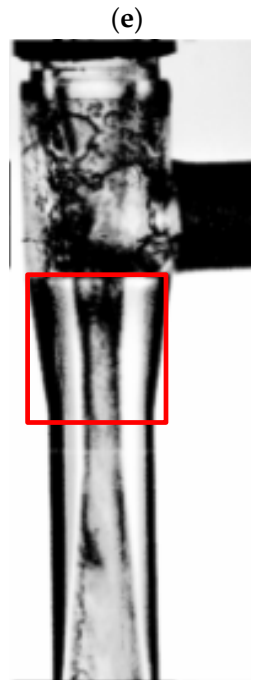

(j)

Figure 11. Gas-liquid mixing pulsating phenomenon of suction chamber images at critical point. (a) 47 ms; (b) $48 \mathrm{~ms}$; (c) 49 ms; (d) $50 \mathrm{~ms}$; (e) $51 \mathrm{~ms}$; (f) $52 \mathrm{~ms}$; (g) $53 \mathrm{~ms}$; (h) $54 \mathrm{~ms}$; (i) $55 \mathrm{~ms}$; (j) $56 \mathrm{~ms}$.

Figure 12 shows the proportion of shadow part (secondary flow) in the red square, and it can be concluded that the pulsation phenomenon has a certain periodicity of approximately $10 \mathrm{~ms}$. The periodic pulsation of the gas-liquid flow and the spray half cone-angle oscillation are directly related to the large deformation of the gas-liquid mixture entering the constant pressure mixing section. The complex mixed flow field structure in the primary nozzle and the suction chamber also directly 
causes these conditions to occur. The large amount of secondary flow entering the mixing chamber from the suction chamber hinders the injection of the primary flow, the decrease in the primary flow leads to reduced secondary flow and obstruction, and the primary flow increases again, which in turn causes more secondary flow to enter the mixing chamber. The increase in secondary flow impedes the primary flow again, resulting in the pulsation phenomenon. Figure 13 shows the correlation between pulsating and ER, and their frequency distribution histogram is shown in Figure 14. It can be seen from the figure that the pulsation phenomenon affects the fluctuation of the ER. Although the amplitude change is small, enough attention should be paid to it. Therefore, how to reduce the occurrence of pulsation phenomenon is of great significance in practical engineering.

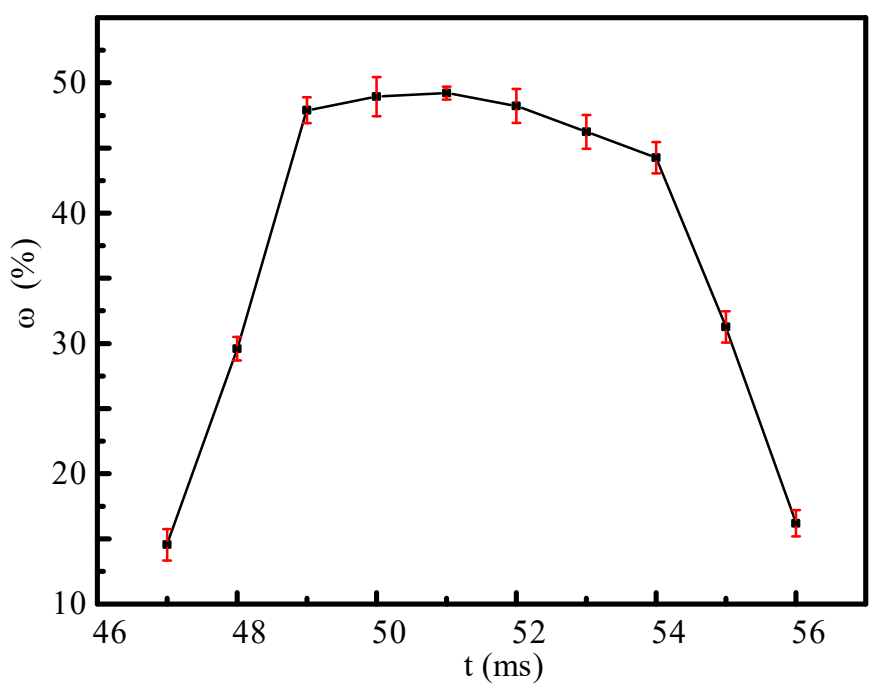

Figure 12. Proportion of secondary flow in the red square.

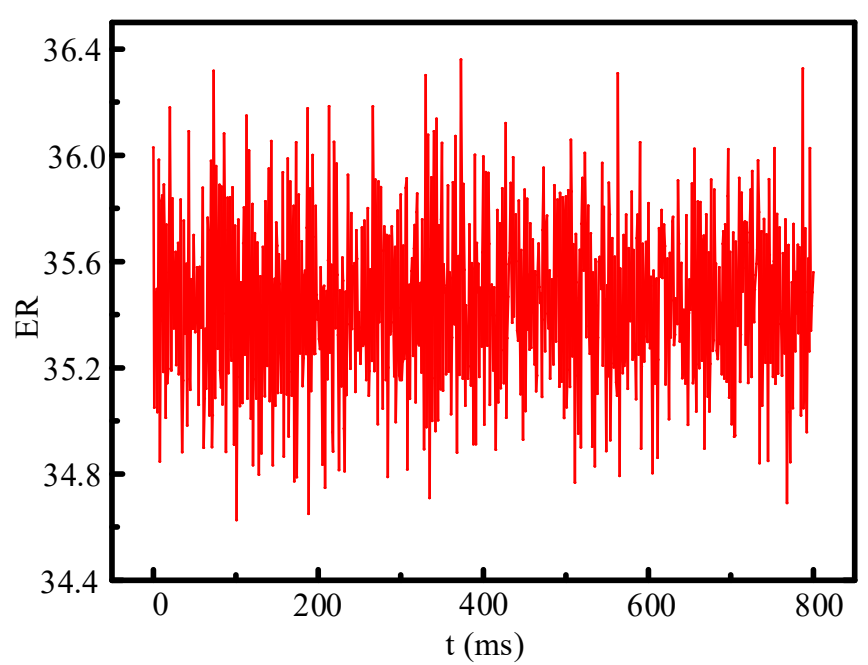

Figure 13. The correlation between pulsating and entrainment ratio (ER). 


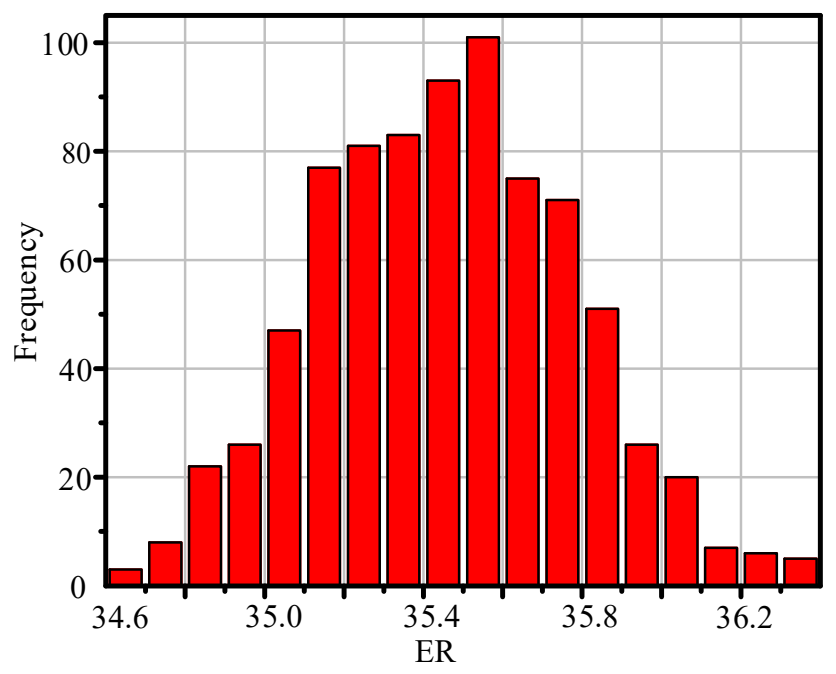

Figure 14. Frequency distribution histogram.

\section{Conclusions}

A two-phase flow ejector is very important for improving the efficiency of a water mist fire suppression system. This paper proposes an ejector suitable for a water mist fire suppression system and aims to improve the efficiency of the whole system by studying the ejector. The effects of the primary flow pressure and different ejector structures on the ejector performance were discussed. By means of a high-speed camera, the atomization state and the two-phase flow pulsation phenomenon of the mixing section were photographed. According to the experimental results and discussion, the following conclusions can be drawn:

- When selecting the structure parameters of the ejector, the optimal $P_{\mathrm{P}}$ range can be adjusted by the difference between the ARs, and the overall performance of the ejector can be improved by adjusting the distance of the NXP to improve the efficiency of the water mist fire suppression system. The ejector with an AR of 6 demonstrated the best performance.

- With increasing $P_{\mathrm{P}}$, the ER of the ejector tends to first increase and then decrease because $m_{\mathrm{p}}$ increases as the primary flow pressure increases, but $m_{\mathrm{S}}$ decreases rapidly beyond the optimum operating point of the ejector.

- The larger the AR is, the larger the ER of the ejector. The maximum ER of the AR6 ejector is 28.07. An increase in the NXP also leads to an increase in the ER. The ejector with an NXP of +1 demonstrated the best performance, and the ER of this ejector reached 36.29.

- The atomization effect of the ejector and the spray half cone-angle increase as the ER increases. The maximum spray half cone-angle is $7.07^{\circ}$. When the ejector passes the optimum operating point $\left(P_{\mathrm{P}}\right.$ is $\left.3.6 \mathrm{bar}\right)$, a small portion of air is drawn into the ejector due to the pressure difference at that time, resulting in a reduction in the atomization effect and a decrease in the spray half cone-angle. The spray half cone-angle is unstable mainly due to the two-phase flow pulsation phenomenon when the ejector is working. The pulsation period of the ejector employed in the experiment is approximately $10 \mathrm{~ms}$. The complex mixed flow field structure in the primary nozzle and the suction chamber also directly cause these conditions to occur.

Author Contributions: Conceptualization, S.L. and J.Z.; Data curation, W.L. and S.L.; Investigation, W.L. and J.Z.; Software, S.L. and J.Z.; Writing—original draft, W.L.; Writing—review and editing, J.Z. and C.J. Funding acquisition, Y.L., S.L., and J.Z. All authors have read and agreed to the published version of the manuscript.

Funding: This research was funded by the National Natural Science Foundation of China (Grant No. 51705288), Shandong Provincial Natural Science Foundation (ZR2017QEE001, 2018GHY115006, 2019GSF109051), National Key R\&D Program of China (2016YFE0205700), and Open Foundation of the State Key Laboratory of Fluid Power and Mechatronic Systems, China (GZKF-201805). 
Conflicts of Interest: The authors declare no conflicts of interest.

\section{Nomenclature}

$Q_{G} \quad$ Gas flow rate $\left(\mathrm{m}^{3} / \mathrm{s}\right)$

$Q_{\mathrm{L}} \quad$ Liquid flow rate $\left(\mathrm{m}^{3} / \mathrm{s}\right)$

$d_{1} \quad$ Diameter of primary nozzle inlet $(\mathrm{mm})$

$D_{\mathrm{n}} \quad$ Diameter of mixing chamber inlet $(\mathrm{mm})$

$D_{\mathrm{m}} \quad$ Diameter of constant-area mixing chamber $(\mathrm{mm})$

$D_{\mathrm{f}} \quad$ Diameter of diffuser outlet $(\mathrm{mm})$

$D_{\mathrm{s}} \quad$ Diameter of secondary flow chamber $(\mathrm{mm})$

$L_{\mathrm{p}} \quad$ Length of primary nozzle $(\mathrm{mm})$

$L_{\mathrm{n}} \quad$ Length of constant-pressure mixing chamber $(\mathrm{mm})$

$L_{\mathrm{m}} \quad$ Length of constant-area mixing chamber $(\mathrm{mm})$

$L_{\mathrm{d}} \quad$ Length of diffuser ( $\mathrm{mm}$ )

$P_{\mathrm{p}} \quad$ Primary flow pressure (bar)

$P_{\mathrm{s}} \quad$ Secondary flow pressure (bar)

$P_{\mathrm{b}} \quad$ Back pressure (bar)

$P_{\mathrm{b}}^{*} \quad$ Critical pressure (bar)

$m_{\mathrm{p}} \quad$ Primary flow mass flow rate $(\mathrm{g} / \mathrm{s})$

$m_{\mathrm{S}} \quad$ Secondary flow mass flow rate $(\mathrm{g} / \mathrm{s})$

$\theta \quad$ Spray half cone-angle (degree)

$\omega \quad$ Proportion of secondary flow (\%)

$E R \quad$ Entrainment ratio

$A R \quad$ Ejector area ratio

NXP Nozzle exit position (mm)

LED Light-emitting diode

\section{References}

1. Keenan, J.H. A Simple Air Ejector. J. Appl. Mech. Trans. ASME 1942, 64, 75-81.

2. Keenan, J.H.; Neumann, E.P.; Lustwerk, F. An investigation of ejector design by analysis and experiment. J. Appl. Mech. Trans. ASME 1950, 72, 299-309.

3. Sun, D.W.; Eames, I.W. Recent developments in the design theories and applications of ejectors-A review. J. Inst. Energy 1995, 68, 65-79.

4. Zhou, Y.; Peng, S.; Huang, X.; Wu, C.; Zhang, J. Lng-air mixture as a supplementary energy injection into a biogas distribution network. Energies 2017, 10, 1902. [CrossRef]

5. Chandra, V.V.; Ahmed, M.R. Experimental and computational studies on a steam jet refrigeration system with constant area and variable area ejectors. Energy Convers. Manag. 2014, 79, 377-386. [CrossRef]

6. Hu, J.; Shi, J.; Liang, Y.; Yang, Z.; Chen, J. Numerical and experimental investigation on nozzle parameters for R410A ejector air conditioning system. Int. J. Refrig. 2014, 40, 338-346. [CrossRef]

7. Taslimi Taleghani, S.; Sorin, M.; Poncet, S. Analysis and optimization of exergy flows inside a transcritical $\mathrm{CO}_{2}$ ejector for refrigeration, air conditioning and heat pump cycles. Energies 2019, 12, 1686. [CrossRef]

8. Nakagawa, M.; Marasigan, A.R.; Matsukawa, T.; Kurashina, A. Experimental investigation on the effect of mixing length on the performance of two-phase ejector for $\mathrm{CO}_{2}$ refrigeration cycle with and without heat exchanger. Int. J. Refrig. 2011, 3, 1604-1613. [CrossRef]

9. Liu, F.; Groll, E.A.; Li, D. Investigation on performance of variable geometry ejectors for $\mathrm{CO}_{2}$ refrigeration cycles. Energy 2012, 45, 829-839. [CrossRef]

10. Dong, J.; Wang, W.; Han, Z.; Ma, H.; Deng, Y.; Su, F.; Pan, X. Experimental investigation of the steam ejector in a single-effect thermal vapor compression desalination system driven by a low-temperature heat source. Energies 2018, 11, 2282. [CrossRef]

11. Yan, J.; Wen, N.; Wang, L.; Li, X.; Liu, Z.; Li, S. Optimization on ejector key geometries of a two-stage ejector-based multi-evaporator refrigeration system. Energy Convers. Manag. 2018, 175, 142-150. [CrossRef]

12. Thongtip, T.; Aphornratana, S. An experimental analysis of the impact of primary nozzle geometries on the ejector performance used in R141b ejector refrigerator. Appl. Therm. Eng. 2017, 110, 89-101. [CrossRef] 
13. Li, C.; Yan, J.; Li, Y.; Cai, W.; Lin, C.; Chen, H. Experimental study on a multi-evaporator refrigeration system with variable area ratio ejector. Appl. Therm. Eng. 2016, 102, 196-203. [CrossRef]

14. Wang, L.; Yan, J.; Wang, C.; Li, X. Numerical study on optimization of ejector primary nozzle geometries. Int. J. Refrig. 2017, 76, 219-229. [CrossRef]

15. Cui, J.; Lai, H.; Feng, K.; Ma, Y. Quantitative analysis of the minor deviations in nozzle internal geometry effect on the cavitating flow. Exp. Therm. Fluid Sci. 2018, 94, 89-98. [CrossRef]

16. Sriveerakul, T.; Aphornratana, S.; Chunnanond, K. Performance prediction of steam ejector using computational fluid dynamics: Part 1. Validation of the CFD results. Int. J. Therm. Sci. 2007, 46, 812-822. [CrossRef]

17. Chen, J.; Jarall, S.; Havtun, H.; Palm, B. A review on versatile ejector applications in refrigeration systems. Renew. Sustain. Energy Rev. 2015, 49, 67-90. [CrossRef]

18. Mahrukh, M.; Kumar, A.; Gu, S. Effects of angular injection, and effervescent atomization on high-velocity suspension flame spray process. Surf. Coat. Technol. 2016, 302, 368-382. [CrossRef]

19. Sen, D.; Balzan, M.A.; Nobes, D.S.; Fleck, B.A. Bubble formation and flow instability in an effervescent atomizer. J. Vis. 2014, 17, 113-122. [CrossRef]

20. Maldonado, S.; Fleck, B.; Heidrick, T.; Amirfazli, A.; Chan, E.W.; Knapper, B. Development of an experimental method to evaluate the stability of gas-liquid sprays. At. Sprays 2008, 18, 699-722. [CrossRef]

21. Lin, K.-C.; Kennedy, P.; Jackson, T. Structures of internal flow and the corresponding spray for aerated-liquid injectors. In Proceedings of the 37th AIAA/ASME/SAE/ASEE Joint Propulsion Conference, Salt Lake City, UT, USA, 8-11 July 2001.

22. Tratnig, A.; Brenn, G. Drop size spectra in sprays from pressure-swirl atomizers. Int. J. Multiph. Flow 2010, 36, 349-363. [CrossRef]

23. Yang, L.-J.; Fu, Q.-F.; Zhang, W.; Du, M.-L.; Tong, M.-X. Spray characteristics of gelled propellants in novel impinging jet injector. J. Propuls. Power 2013, 29, 104-113. [CrossRef]

24. Martínez-Galván, E.; Antón, R.; Ramos, J.C.; Khodabandeh, R. Effect of the spray cone angle in the spray cooling with R134a. Exp. Therm. Fluid Sci. 2013, 50, 127-138. [CrossRef]

25. Jia, T.-M.; Yu, Y.-S.; Li, G.-X. Experimental investigation of effects of super high injection pressure on diesel spray and induced shock waves characteristics. Exp. Therm. Fluid Sci. 2017, 85, 399-408. [CrossRef]

26. Esfarjani, S.A.; Dolatabadi, A. A 3D simulation of two-phase flow in an effervescent atomizer for suspension plasma spray. Surf. Coat. Technol. 2009, 203, 2074-2080. [CrossRef]

27. Zhu, Y.; Wang, Z.; Yang, Y.; Jiang, P.-X. Flow visualization of supersonic two-phase transcritical flow of $\mathrm{CO}_{2}$ in an ejector of a refrigeration system. Int. J. Refrig. 2017, 74, 354-361. [CrossRef]

28. Zhu, Y.; Li, Y. Novel ejector model for performance evaluation on both dry and wet vapors ejectors. Int. J. Refrig. 2009, 32, 21-31. [CrossRef]

29. Wang, L.; Liu, J.; Zou, T.; Du, J.; Jia, F. Auto-tuning ejector for refrigeration system. Energy 2018, 161, $536-543$. [CrossRef]

30. Dong, J.M.; Song, H.; Yu, M.Q.; Wang, W.N.; Pan, X.X. Numerical investigation of miniature ejector refrigeration system embedded with a capillary pump loop. Micromachines 2017, 8, 235. [CrossRef]

31. Han, Y.; Wang, X.; Sun, H.; Zhang, G.; Guo, L.; Tu, J. CFD simulation on the boundary layer separation in the steam ejector and its influence on the pumping performance. Energy 2019, 167, 469-483. [CrossRef]

32. Zhang, X.; He, Z.; Wang, Q.; Tao, X.; Zhou, Z.; Xia, X.; Zhang, W. Effect of fuel temperature on cavitation flow inside vertical multi-hole nozzles and spray characteristics with different nozzle geometries. Exp. Therm. Fluid Sci. 2018, 91, 374-387. [CrossRef]

33. Chen, S.K.; Lefebvre, A.H. Discharge coefficients for plain-orifice effervescent atomizers. At. Sprays 1994, 4, 275-290. [CrossRef]

(C) 2020 by the authors. Licensee MDPI, Basel, Switzerland. This article is an open access article distributed under the terms and conditions of the Creative Commons Attribution (CC BY) license (http://creativecommons.org/licenses/by/4.0/). 